\title{
RESPUESTA DE LA MORERA (Morus alba) A LA FERTILIZACIÓN NITROGENADA, DENSIDADES DE SIEMBRA Y A LA DEFOLIACIÓN 1
}

\author{
Carlos Boschini F.', Herbert Dormond H.2. Alvaro Castro R. ${ }^{3}$
}

\begin{abstract}
RESUMEN
Respuesta de la morera (Morus alba) a la fertilización nitrogenada, densidades de siembra y a la defoliación. En la Estación Experimental de Ganado Lechero "Alfredo Volio Mata", ubicada en una zona de bosque húmedo montano bajo, se evaluó una plantación de morera de dos años de edad, dividida en tres parcelas, con un distanciamiento entre plantas de 60,90 y $120 \mathrm{~cm}$ entre hileras y plantas. Cada parcela se uniformó en una altura de poda de $60 \mathrm{~cm}$. Las subparcelas se podaron en forma consecutiva a 56,84 y 112 días durante 224 días. Se adicionaron cinco dosis de nitrógeno ( 0 , $150,300,450$ y $600 \mathrm{~kg} / \mathrm{ha})$. El rendimiento anual de materia seca de la planta entera de morera, sus tallos y hojas, resultó significativo $(\mathrm{P}=0,01)$ influido por la distancia de siembra y la frecuencia de corte. El rendimiento de hojas y tallos disminuyó conforme aumentó la distancia de siembra. La frecuencia de corte mostró una influencia marcada $(\mathrm{P}=0,01)$ sobre la producción de biomasa en la planta entera y sus partes. El nitrógeno no mostró ningún efecto diferencial $(\mathrm{P}>0,05)$ sobre el rendimiento de biomasa total o sobre la producción de hojas o tallos.
\end{abstract}

\begin{abstract}
Mulberry bush (Morus alba) response to nitrogen fertilization, sowing densities and to defoliation. This experiment was carried out at the Alfredo Volio Mata Dairy Cattle Experimental Station in Costa Rica. A mulberry bush plantation was divided into three plots: with a distance of 60 , 90 and $120 \mathrm{~cm}$ between rows and plants. All plots were uniformly pruned at $60 \mathrm{~cm}$ from the ground. During a 224-day period, these subplots were pruned consecutively every 56th, 84th and 112th day. The annual yield of dry matter of the whole mulberry bush (stems and leaves) was influenced ( $\mathrm{P}=$ 0.01 ) by all the factors studied. The planting distance had a decreasing effect on the Yield of stems and leaves increased as the distance decreased. The cutting frequency had a marked influence $(\mathrm{P}=0.01)$ on the production of biomass in the whole plant and its parts. A linear effect $(\mathrm{P}=0.01)$ was observed on the stem yield, as well as the whole plant, when the cutting interval increased between the 56th and 112th days. A negative effect $(\mathrm{P}<0.05)$ was observed in the leaf:stem ratio when the pruning interval was increased. The nitrogen dosage used in the experiment $(0,150,300,450$ and $600 \mathrm{~kg} / \mathrm{ha})$ did not show a differential effect $(\mathrm{P}<0.05)$ on the total biomass yield nor on the stem or leaf production. No significant effects were observed between the planting distance or the cutting frequency and the nitrogen dosage.
\end{abstract}

\section{INTRODUCCIÓN}

La morera se ha adaptado en Mesoamérica, especialmente para la producción de follaje empleado en la alimentación de bovinos y caprinos (Rodríguez, Arias y Quiñonez, 1994; González, 1996). Es una especie arbórea que en condiciones de crecimiento libre alcanza los 12 metros de altura. Se comporta como una arbustiva perenne bajo un manejo de poda periódica. Es una planta de crecimiento rápido, con un sistema radical fuerte, profuso y de rebrotes foliares vigorosos (Paolieri, 1970; Tieng-Zing et al., 1988).
La producción de biomasa en explotación intensiva, sembrada en suelos bien drenados, zonas de alta luminosidad y con una precipitación media o alta, muestra en Costa Rica rendimientos de biomasa fresca de 70-119 t/ha/año (Boschini, Dormond y Castro, 1998; Espinoza, 1996). En Centroamérica, se han informado de rendimientos de 19 a $28 \mathrm{t} / \mathrm{ha} / \mathrm{año}$ de materia seca con plantas enteras, cosechadas a $60-80 \mathrm{~cm}$ sobre el nivel del suelo, en intervalos de poda de seis a 12 semanas y con densidades de siembra de 20000 plantas por hectárea (Blanco, 1992; Rodríguez, Arias y Quiñonez, 1994). Los resultados de los experimentos en

\footnotetext{
1 Inscrito en la Vicerrectoría de Investigación, No 737-96-303. Universidad de Costa Rica.

2 Estación Experimental Alfredo Volio Mata, Facultad de Agronomía, Universidad de Costa Rica.

3 Programa de Ganado Caprino. Ministerio de Agricultura y Ganadería. Gobierno de Costa Rica.
} 
Mesoamérica muestran una tendencia a que el rendimiento por área mejora al aumentar la densidad de siembra y el plazo entre podas. La producción foliar es superior a la del tallo durante los primeros 100 días de rebrote (Boschini, Dormond y Castro, 1998), posteriormente se favorece la producción de tallo. La calidad bromatológica de las hojas, particularmente el contenido de proteína, se mantiene constante durante los primeros cuatro a cinco meses (Estrada, 1995; Benavides, Lachaux y Fuentes, 1994; Velazquez et al., 1993).

Los altos contenidos de proteína en las hojas y los grandes rendimientos de biomasa por unidad de área, indican la necesidad de reponer al suelo el nitrógeno extraído por la planta, en proporciones relativamente altas. La Asociación Internacional de Industriales de Fertilizantes recomiendan 300, 160 y $200 \mathrm{~kg} / \mathrm{ha} /$ año de $\mathrm{N}, \mathrm{P}_{2} \mathrm{O}_{5}$ y K $\mathrm{K}_{2} \mathrm{O}$ respectivamente (IFA, 1992) en suelos de origen volcánico. Rodríguez, Arias y Quiñonez (1994) han aplicado dosis de cero, 40 y $80 \mathrm{~kg}$ de N/ha/corte en Guatemala, con rendimientos aceptables, pero observaron una baja respuesta en el incremento de proteína cruda en las hojas (17,5 a 18\%). Benavides, Lachaux y Fuentes, (1994) en Costa Rica, empleó niveles de fertilización nitrogenada de cero a $480 \mathrm{~kg}-$ /ha/año, durante tres años, logrando rendimientos medios de 19 a 30 toneladas de materia seca por ha/año. La morera ha mostrado, en condiciones tropicales, altos rendimientos de materia seca a través de los ciclos anuales de producción. Se hace necesario conocer la respuesta del cultivo de morera a la fertilización nitrogenada bajo diferentes prácticas de establecimiento y poda. El experimento de campo se llevó a cabo con el propósito de conocer el efecto de diferentes dosis de nitrógeno, aplicado al suelo, sobre la producción de biomasa, en cultivos sembrados a diferentes distancias entre plantas y cosechados a diferentes frecuencias.

\section{MATERIALES Y MÉTODOS}

El trabajo de campo se llevó a cabo en la Estación Experimental Alfredo Volio Mata, ubicada en la latitud 10 norte, longitud 84 oeste, a $1542 \mathrm{msnm}$ de altitud, $2050 \mathrm{~mm}$ de precipitación media anual, distribuida entre los meses de mayo a noviembre. Los restantes meses son de periodo seco. La temperatura media es de $19,5^{\circ} \mathrm{C}$ y una humedad relativa promedio de $84 \%$. El suelo está clasificado como Typic Distrandepts, de origen volcánico (Vasquez, 1982), caracterizado por tener una profundidad media con buen drenaje natural, una fertilidad media (Cuadro 1). Dentro de la clasificación ecológica, la zona se tipifica como bosque húmedo montano bajo (Tosi, 1970; citado por Vásquez, 1982).

Se empleó una plantación establecida en mayo de 1993 en una área de $5000 \mathrm{~m}^{2}$. Se dividió en tres parcelas grandes con distancias de siembra de 60, 90 y 120 $\mathrm{cm}$ entre hileras y plantas, cada lote. Las densidades de siembra correspondieron a $27.777 ; 12.345$ y 6.944 plantas/ha (Boschini, Dormond y Castro, 1998). En mayo de 1995, se podaron las plantas de los tres lotes, de manera uniforme a $60 \mathrm{~cm}$ de altura sobre el nivel del suelo. A partir de la fecha de uniformización se progra-

Cuadro 1. Análisis químico inicial del suelo sembrado con el cultivo de morera. Cartago, Costa Rica. 1995.

\begin{tabular}{|c|c|c|c|c|c|}
\hline \multirow[b]{2}{*}{ Contenido } & \multirow[b]{2}{*}{ Unidades } & \multicolumn{4}{|c|}{ Profundidad de suelo, $\mathrm{cm}$} \\
\hline & & 0 - 10 & $10-20$ & $20-30$ & Promedio \\
\hline $\mathrm{pH}$ & & 6,10 & 6,20 & 6,20 & 6,17 \\
\hline Materia orgánica & $\%$ & 4,02 & 10,52 & 3,48 & 6,01 \\
\hline Nitrógeno total & $\%$ & 0,23 & 0,46 & 0,19 & 0,29 \\
\hline Nitrógeno - nitrato & $\mathrm{mg} / \mathrm{kg}$ & 41,73 & 10,57 & 10,07 & 20,79 \\
\hline Nitrógeno - amoniacal & $\mathrm{mg} / \mathrm{kg}$ & 4,06 & 2,15 & 5,16 & 3,79 \\
\hline Potasio & $\mathrm{cmol} / \mathrm{l}$ & 1,73 & 1,48 & 1,17 & 1,46 \\
\hline Fósforo & $\mathrm{mg} / \mathrm{l}$ & 16,30 & 12,60 & 12,20 & 13,70 \\
\hline Calcio & $\mathrm{cmol} / \mathrm{l}$ & 8,32 & 6,97 & 7,52 & 7,60 \\
\hline Magnesio & $\mathrm{cmol} / 1$ & 4,11 & 4,01 & 4,13 & 4,08 \\
\hline CICE & & 14,30 & 12,56 & 12,87 & 13,24 \\
\hline Acidez & $\mathrm{cmol} / \mathrm{l}$ & 0,14 & 0,10 & 0,05 & 0,10 \\
\hline Cobre & $\mathrm{mg} / \mathrm{l}$ & 32,50 & 35,90 & 35,40 & 34,60 \\
\hline Hierro & $\mathrm{mg} / \mathrm{l}$ & 323,00 & 364,00 & 370,00 & 352,33 \\
\hline Manganeso & $\mathrm{mg} / \mathrm{l}$ & 15,70 & 8,00 & 7,00 & 10,23 \\
\hline Zinc & $\mathrm{mg} / \mathrm{l}$ & 3,20 & 3,00 & 3,60 & 3,27 \\
\hline Boro & $\mathrm{mg} / \mathrm{l}$ & 0,45 & 0,35 & 0,28 & 0,36 \\
\hline Azufre & $\mathrm{mg} / \mathrm{l}$ & 4,18 & 4,55 & 3,88 & 4,20 \\
\hline
\end{tabular}


maron tres cortes consecutivos cada 56 días, dos cada 84 y 112 días (subparcelas). Cada subparcela fue dividida en cinco subsubparcelas para los tratamientos de fertilización nitrogenada. Cuando el rebrote de las plantas alcanzó de tres a cinco $\mathrm{cm}$ de largo (10-14 días posteriores a la poda) las parcelas pequeñas se fertilizaron con nitrato de amonio, en las dosis correspondientes a los tratamientos cero, $150,300,450$ y $600 \mathrm{~kg} \mathrm{~N} / \mathrm{ha} / \mathrm{año}$.

Se empleó una estructura experimental de parcelas subsubdivididas, con observaciones en el tiempo desigualmente repetidas. En las parcelas grandes se ubicaron las distancias entre plantas, las frecuencias de corte en las subparcelas y en las parcelas pequeñas a los niveles de fertilización nitrogenada, usando el siguiente modelo matemático:

$\mathrm{Y}_{\mathrm{ijk} l m}=\mathrm{U}+\mathrm{D}_{\mathrm{i}}+\mathrm{F}_{\mathrm{j}}+\mathrm{N}_{\mathrm{k}}+\mathrm{C}(\mathrm{F})_{\mathrm{jl}}+\mathrm{DF}_{\mathrm{ij}}+\mathrm{DN}_{\mathrm{ik}}+\mathrm{FN}_{\mathrm{jk}}+\mathrm{DF}-$ $\mathrm{N}_{\mathrm{ijk}}+\mathrm{E}_{\mathrm{ijklm}}$

donde:

$\mathrm{Y}_{\mathrm{ijklm}}=$ Producción de materia seca en $\mathrm{kg} / \mathrm{ha} / \mathrm{año}$.

$\mathrm{U}=$ Media general.

$\mathrm{D}_{\mathrm{i}}=$ Efecto de la i-ésima distancia de siembra $(60,90$, $120 \mathrm{~cm}$ ).

$F_{j}=$ Efecto de la j-ésima frecuencia de corte $(56,84$, 112 días).

$\mathrm{N}_{\mathrm{k}}=$ Efecto del k-ésimo nivel de nitrógeno (cero, 150, $300,450,600 \mathrm{~kg} / \mathrm{ha} / \mathrm{año})$.

$\mathrm{C}(\mathrm{F})_{\mathrm{ij}}=$ Efecto del l-ésimo número de corte dentro de la l-ésima frecuencia de corte.

$\mathrm{DF}_{\mathrm{ij}}=$ Efecto de i-ésima distancia de siembra por la $\mathrm{j}$ ésima frecuencia de corte.

$\mathrm{DN}_{\mathrm{ik}}=$ Efecto de i-ésima distancia de siembra por el késimo nivel de fertilización.

$\mathrm{FN}_{\mathrm{jk}}=$ Efecto de j-ésima frecuencia de corte por el késimo nivel de fertilización.

$\mathrm{DFN}_{\mathrm{ijk}}=$ Efecto de la i-ésima distancia de siembra por la j-ésima frecuencia de corte por el k-ésimo nivel de fertilización.

$\mathrm{E}_{\mathrm{ijklm}}=$ Error experimental.

Cada parcela fue podada completamente, en las frecuencias de corte programadas, a $60 \mathrm{~cm}$ sobre el nivel de suelo, con tijera. La producción de biomasa fresca se pesó en el campo. Posteriormente se obtuvo una muestra del material cosechado y se separaron las hojas y tallos. Cada una fue pesada en fresco, secada a $60{ }^{\circ} \mathrm{C}$ durante 48 horas hasta alcanzar un peso constante y pesada nuevamente en seco. Estas muestras se molieron en un molino Willey, con malla de un $\mathrm{mm}$ de paso. Posteriormente, se determinó el contenido de materia seca a $105^{\circ} \mathrm{C}$ (AOAC, 1980). Se estimaron los rendimientos de biomasa seca, en la planta entera, hojas y tallos, a partir de los muestreos realizados. Los resultados se analizaron con el PROC GLM del paquete estadístico SAS (1985) con el modelo propuesto. A los efectos principales que fueron significativos $(\mathrm{P}<0,05)$ en el análisis de varianza, se les hizo un análisis de tendencias, debido a que los niveles en cada factor estudiado se distribuyeron ortogonalmente.

\section{RESULTADOS}

La producción de materia seca total de morera, sus tallos y hojas, obtenido en este experimento de campo, en cada una de las distancias de siembra, frecuencias de corte y niveles de fertilización nitrogenada se presentan en el Cuadro 2. El rendimiento general de la planta entera fue de 19097 (DE 7790) kg/ha/año de materia seca, de tallo fue 9353 (DE 4809) y de hoja 9744 (DE $3804) \mathrm{kg} / \mathrm{ha} / \mathrm{año}$. Las distancias de siembra y las frecuencias de defoliación resultaron altamente significativas $(\mathrm{P}<0,01)$ sobre la producción de biomasa total de la planta y de sus componentes. El factor niveles de fertilización nitrogenada, como efecto principal, no mostró ninguna influencia importante $(\mathrm{P}>0,05)$ sobre la producción de materia seca en la planta entera, en tallos y hojas.

La densidad de siembra tuvo un marcado efecto sobre la producción de materia seca en las plantas. Conforme aumentó la distancia de siembra, se observó que los rendimientos disminuyeron a tasas decrecientes (tendencia cuadrática). En la distancia de $60 \mathrm{~cm}$ entre plantas, la producción de hojas y tallos fue muy similar. $\mathrm{Al}$ aumentar la distancia de siembra a $90 \mathrm{~cm}$, la producción de hojas disminuyó a razón de 180,9 kg/ha/año por cada $\mathrm{cm}$ en el aumento de la distancia entre hileras y plantas dentro de las hileras. En este mismo intervalo, la producción de tallos disminuyó $215,3 \mathrm{~kg} / \mathrm{ha} /$ año por cada $\mathrm{cm}$ de aumento en la distancia de siembra. Cuando la distancia entre plantas subió a $120 \mathrm{~cm}$, el rendimiento de hojas disminuyó a una tasa de $80,9 \mathrm{~kg} / \mathrm{ha} / \mathrm{a}-$ ño y la de tallos en 74,2 kg/ha/año por cada $\mathrm{cm}$ de aumento en la distancia de siembra entre 90 y $120 \mathrm{~cm}$. La disminución en el rendimiento de la planta entera fue de un $41,6 \%$ al incrementarse la distancia de siembra de 60 a $90 \mathrm{~cm}$ y de $27,9 \%$ al subir de 90 a $120 \mathrm{~cm}$ entre plantas. La relación hoja : tallo fue de 0,$98 ; 1,10$ y 1,10 para las distancias de siembra de 60,90 y 120 
Cuadro 2. Producción anual de materia seca de morera, cosechada a diferentes distancias de siembra, frecuencias de corte y niveles de fertilización nitrogenada en kg/ha. Cartago, Costa Rica. 1995.

\begin{tabular}{|c|c|c|c|c|c|c|}
\hline Variable & $\begin{array}{c}\text { Promedio } \\
\text { Planta entera }\end{array}$ & DE & $\begin{array}{l}\text { Promedio } \\
\text { Tallos }\end{array}$ & DE & $\begin{array}{l}\text { Promedio } \\
\text { Hojas }\end{array}$ & DE \\
\hline \multicolumn{7}{|c|}{$\begin{array}{l}\text { Distancias de siembra } \\
\text { cm }\end{array}$} \\
\hline 60 & 28574 & 13034 & 14401 & 6011 & 14172 & 6432 \\
\hline 90 & 16686 & 7024 & 7942 & 4779 & 8744 & 3507 \\
\hline 120 & 12033 & 6790 & 5716 & 3619 & 6316 & 3768 \\
\hline \multicolumn{7}{|c|}{$\begin{array}{l}\text { Frecuencias de corte } \\
\text { (días) }\end{array}$} \\
\hline 56 & 12949 & 7579 & 5524 & 3281 & 7425 & 3345 \\
\hline 84 & 20414 & 9530 & 9709 & 4977 & 10705 & 5002 \\
\hline 112 & 27004 & 14160 & 14741 & 7646 & 12261 & 5752 \\
\hline \multicolumn{7}{|c|}{ Niveles de $\mathbf{N}$ (kg/ha/año) } \\
\hline 0 & 16676 & 7027 & 7726 & 4426 & 8949 & 4880 \\
\hline 150 & 19663 & 6526 & 9875 & 5513 & 9788 & 4265 \\
\hline 300 & 19726 & 7721 & 9694 & 5212 & 10032 & 5835 \\
\hline 450 & 20341 & 8594 & 9879 & 5442 & 10461 & 5625 \\
\hline 600 & 19082 & 6389 & 9592 & 4548 & 9691 & 4076 \\
\hline
\end{tabular}

$\mathrm{cm}$, respectivamente.

La frecuencia de corte mostró una respuesta diferencial marcada $(\mathrm{P}<0,01)$ sobre el rendimiento de hojas y tallos. Al aumentarse el intervalo periódico de corte, la producción de materia seca incrementó proporcionalmente en la planta entera. En la hoja, el rendimiento entre 56 y 84 días creció a razón de $117 \mathrm{~kg} / \mathrm{ha}$ /día y entre 84 y 112 días fue de 55,6 kg/ha/día. El tallo creció a una velocidad de $149,3 \mathrm{~kg} / \mathrm{ha} /$ día entre las frecuencias de 84 y 112 días. En las dos primeras frecuencias de corte, la producción de hoja fue superior a la de tallo, y en la frecuencia de 112 días esta tendencia se invirtió. Se observa evidentemente una interacción entre las proporciones de hojas y tallos, con valores de relación de 1,34; 1,10 y 0,83 en las frecuencias de 56, 84 y 112 días, respectivamente. Se estimó que la producción de tallo alcanzó la producción de hoja a los 98 días de rebrote, en ese momento la relación hoja:tallo fue igual a uno. Posteriormente, la acumulación de tallo es superior a la de hoja. A pesar de los cambios en las proporciones de hojas y tallos, el rendimiento de materia seca anual por unidad de área, en la planta entera, mostró una tendencia lineal bien definida, compensándose la producción de materia seca en las hojas, de modo inverso, con la producción de tallo.

Los niveles de nitrógeno introducidos al experimento como una fuente de variación, no expresaron diferencias significativas $(P>0,05)$ de orden estadístico o de manejo productivo de consideración importante.
La producción de biomasa total mostró una ligera tendencia de rendimientos decrecientes a través de los cinco niveles de fertilización, con aumentos decrecientes a partir de la aplicación de $150 \mathrm{~kg}$ de N/ha/año, hasta alcanzar un máximo en $450 \mathrm{~kg}$ de N/ha/año y un leve decrecimiento en la producción con la dosis de $600 \mathrm{~kg}$ de N/ha/año. Similar respuesta se observó en la producción de hojas y tallos separadamente. La mayor relación hoja:tallo $(1,15)$ se expresó en el tratamiento de cero fertilización nitrogenada. El nivel de $150 \mathrm{~kg}$ de $\mathrm{N} /$ ha/año estimuló por igual la producción de hoja y tallo $(0,99)$ y a partir de $300 \mathrm{~kg}$ de N/ha/año se observó un incremento de $3,5 \%$ de hoja y un $5,9 \%$ en el nivel siguiente. Con la dosis de $600 \mathrm{~kg}$ de N/ha/año volvió a reducirse esa relación a 1,01 .

La interacción de las fuentes de variación distancia de siembra por frecuencias de corte (Cuadro 3) mostró un efecto significativo sobre la producción de la planta entera $(\mathrm{P}<0,05)$, tallo $(\mathrm{P}<0,01)$ y hoja $(\mathrm{P}<0,05)$. En la Figura 1, se observa el comportamiento del crecimiento de la planta entera en las tres frecuencias de corte dentro de cada distancia de siembra. En la densidad intermedia (90 cm entre plantas) la producción de biomasa total tuvo un comportamiento lineal muy definido a través de las frecuencias de poda aplicadas. Esta tendencia cambió en las distancias de 60 y $120 \mathrm{~cm}$ entre plantas. En la primera de estas dos, el rendimiento se mostró creciente, mientras que a $120 \mathrm{~cm}$ se observó en modo decreciente, conforme aumentó el largo del período de corte. 
Cuadro 3. Producción anual de materia seca de morera, a diferentes distancias de siembra por frecuencia de corte en $\mathrm{kg} / \mathrm{ha}$. Cartago, Costa Rica. 1995.

\begin{tabular}{|c|c|c|c|c|c|c|c|}
\hline \multicolumn{2}{|c|}{ Variable } & \multirow{2}{*}{$\begin{array}{c}\text { Promedio } \\
\text { Planta entera }\end{array}$} & \multirow[t]{2}{*}{ DE } & \multirow{2}{*}{$\begin{array}{l}\text { Promedio } \\
\text { Tallos }\end{array}$} & \multirow[t]{2}{*}{ DE } & \multirow{2}{*}{$\begin{array}{c}\text { Promedio } \\
\text { Hojas }\end{array}$} & \multirow[t]{2}{*}{ DE } \\
\hline $\begin{array}{l}\text { Distancias de } \\
\text { siembra }(\mathrm{cm})\end{array}$ & $\begin{array}{c}\text { Frecuencias } \\
\text { de corte (días) }\end{array}$ & & & & & & \\
\hline 60 & 56 & 19348 & 11599 & 8267 & 5454 & 11082 & 6174 \\
\hline 60 & 84 & 27172 & 8895 & 12972 & 4606 & 14200 & 4412 \\
\hline 60 & 112 & 42813 & 22848 & 25031 & 12423 & 18780 & 10525 \\
\hline 90 & 56 & 11685 & 5485 & 4905 & 2713 & 6779 & 2832 \\
\hline 90 & 84 & 17851 & 2421 & 8747 & 1395 & 9103 & 1073 \\
\hline 90 & 112 & 23023 & 9441 & 11692 & 4920 & 4332 & 4955 \\
\hline 120 & 56 & 7813 & 4056 & 3398 & 1973 & 4414 & 2096 \\
\hline 120 & 84 & 16219 & 3656 & 7408 & 2621 & 8811 & 2695 \\
\hline 120 & 112 & 14174 & 3491 & 7500 & 1965 & 6673 & 1657 \\
\hline
\end{tabular}

En la Figura 2 se presenta el rendimiento de hoja desagregado en los dos factores estudiados. La producción foliar en la distancia de siembra de $60 \mathrm{~cm}$ fue mayor que la observada a $90 \mathrm{~cm}$, sin embargo, ambas muestran un crecimiento lineal conforme aumenta el intervalo de corte. A $120 \mathrm{~cm}$ de distancia de siembra entre plantas, el rendimiento de hoja es menor que los anteriores, en cualquier frecuencia de poda. La tasa de crecimiento declinó después de la frecuencia de 84 días, a razón de $-76,3 \mathrm{~kg}$ de materia seca/ha/día. la productividad de cada planta varió con la densidad de siembra. En la distancia de $60 \mathrm{~cm}$ entre plantas, se observaron rendimientos de 0,$39 ; 0,51$ y $0,67 \mathrm{~kg} / \mathrm{planta} / \mathrm{a}-$ ño a 56, 84 y 112 días de intervalo de poda. A $90 \mathrm{~cm}$ fueron de 0,$55 ; 0,73$ y $0,92 \mathrm{~kg} /$ planta/año y a $120 \mathrm{~cm}$ de

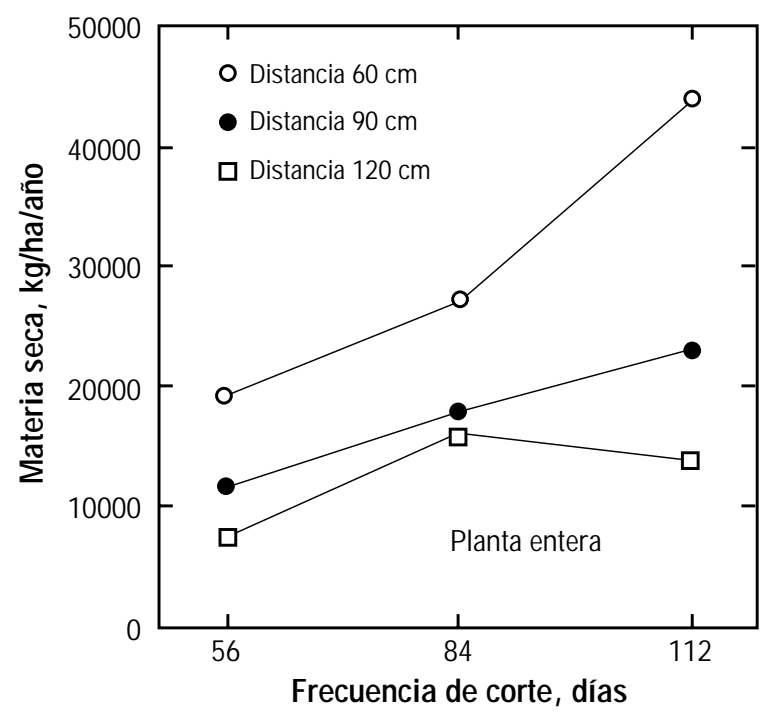

Figura 1. Rendimiento de materia seca de la planta entera de morera, sembrada a tres distancias de siembra y a tres frecuencias de poda. Cartago, Costa Rica. 1995.
0,$63 ; 1,27$ y $0,96 \mathrm{~kg} /$ planta/año en las frecuencias de 56, 84 y 112 días, respectivamente. La Figura 3 muestra el crecimiento del tallo durante los intervalos de corte en cada distancia de siembra. La producción de tallo en la distancia de $60 \mathrm{~cm}$ fue superior que a $90 \mathrm{~cm}$ y esta mayor que a $120 \mathrm{~cm}$ en todas las frecuencias de corte. A $90 \mathrm{~cm}$ entre plantas, el crecimiento es claramente lineal conforme transcurre el intervalo de poda. La velocidad de crecimiento entre 56 y 84 días fue de 168,$0 ; 137,2$ y $143,2 \mathrm{~kg}$ diarios/ha para los distanciamientos de 60,90 y $120 \mathrm{~cm}$. Entre 84 y 112 días, esa tasa mostró variaciones de 430,6; 105,1 y 3,3 kg/ha/día, respectivamente en las distancias de 60,90 y $120 \mathrm{~cm}$. Obviamente las respuestas en ambos periodos fue diferente. El crecimiento diario declinó fuertemente en el

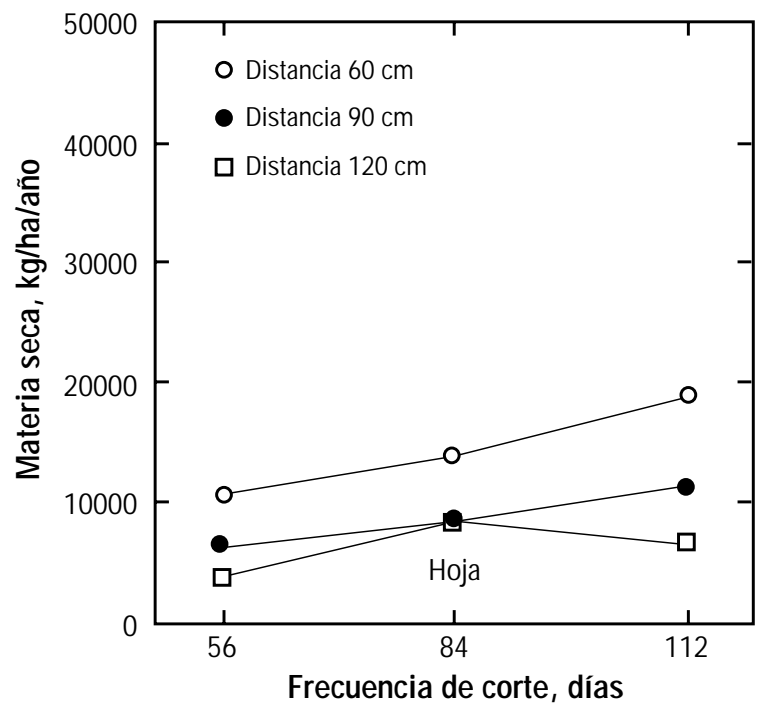

Figura 2. Rendimiento de hoja de morera, sembrada a tres distancias de siembra y a tres frecuencias de poda. Cartago, Costa Rica. 1995. 


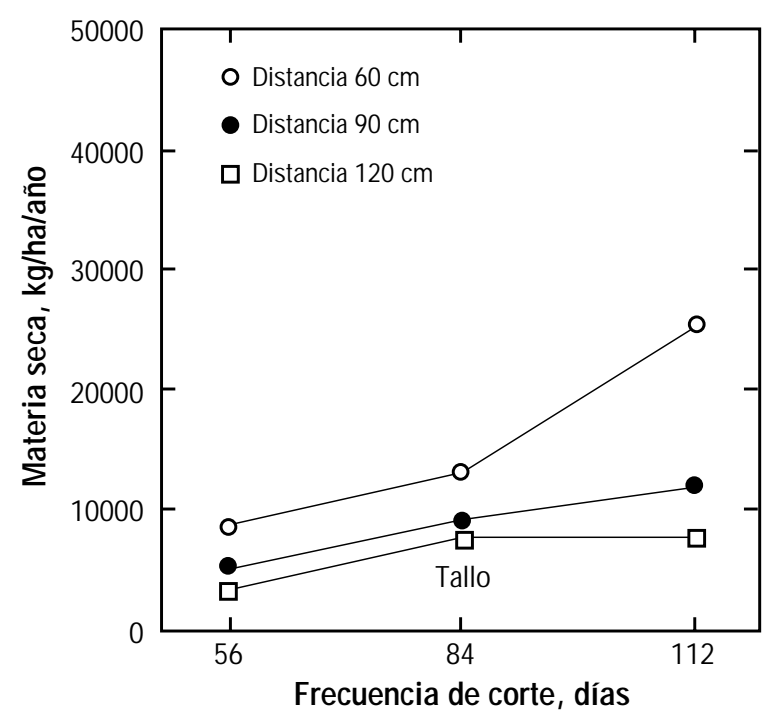

Figura 3. Rendimiento de tallo de morera, sembrada a tres distancias de siembra y a tres frecuencias de poda. Cartago, Costa Rica. 1995.

segundo periodo en la distancia de $120 \mathrm{~cm}$ e incrementó en la distancia de $60 \mathrm{~cm}$. Dentro de cada densidad de siembra, el rendimiento medio de materia seca de tallo fue de 0,$29 ; 0,46$ y $0,90 \mathrm{~kg} / \mathrm{planta} /$ año en las frecuencias de poda de de 56, 84 y 112 días en la distancia de siembra a $60 \mathrm{~cm}$ a $90 \mathrm{~cm}$, fue de 0,$39 ; 0,70$ y $0,94 \mathrm{~kg} / \mathrm{planta} / \mathrm{a}-$ ño y a $120 \mathrm{~cm}$ de 0,$49 ; 1,06$ y $1,08 \mathrm{~kg} /$ planta/año en las frecuencias de 56, 84 y 112 días, respectivamente.
En el Cuadro 4 se presenta la producción anual de materia seca total, de hoja y de tallo, obtenida por interacción de los factores distancia de siembra y dosis de nitrógeno aplicado. En el Cuadro 5 se observan los valores medios de la producción anual de materia seca por hectárea, de la planta entera y sus partes, sometida a tres frecuencias de corte y cinco niveles de fertilización. Tanto la producción de hojas y tallos separadamente como el rendimiento conjunto no mostraron una respuesta diferencial $(\mathrm{P}>0,05)$ a los niveles de fertilizante aplicado en combinación con las distancias de siembra o las frecuencias de defoliación.

La interacción de las variables distancia de siembra por frecuencias de corte y por niveles de fertilización resultó altamente significativa $(\mathrm{P}<0,01)$ para la producción de materia seca total, no significativa $(\mathrm{P}>0,05)$ para la producción de hoja y altamente importante $(\mathrm{P}<$ $0,01)$ para el crecimiento del tallo. Estos resultados presentaron variaciones muy amplias y discontinuas, sin mostrar una tendencia específica particularmente entre los tratamientos de fertilización aplicados.

\section{DISCUSIÓN}

Los resultados experimentales presentados en el Cuadro 2, muestran rendimientos medios generales de morera en la planta entera, hojas y tallos muy similares a los reportados por Boschini et al. (1998) en la misma plantación con dos años menos de edad y manejados

Cuadro 4. Producción anual de materia seca de morera a diferentes distancias de siembra por niveles de fertilización nitrogenada en kg/ha. Cartago, Costa Rica. 1995.

\begin{tabular}{|c|c|c|c|c|c|c|c|}
\hline \multicolumn{2}{|c|}{ Variable } & \multirow{2}{*}{$\begin{array}{c}\text { Promedio } \\
\text { Planta entera }\end{array}$} & \multirow[t]{2}{*}{ DE } & \multirow{2}{*}{$\begin{array}{l}\text { Promedio } \\
\text { Tallos }\end{array}$} & \multirow[t]{2}{*}{ DE } & \multirow{2}{*}{$\begin{array}{l}\text { Promedio } \\
\text { Hojas }\end{array}$} & \multirow[t]{2}{*}{ DE } \\
\hline $\begin{array}{l}\text { Distancias de } \\
\text { siembra(cm) }\end{array}$ & $\begin{array}{c}\text { Fertilizante } \\
\text { nitrogenado } \\
\text { kg/ha/año }\end{array}$ & & & & & & \\
\hline 60 & 0 & 27350 & 17580 & 12948 & 9743 & 14402 & 8076 \\
\hline 60 & 150 & 29464 & 19077 & 15359 & 6600 & 14102 & 9793 \\
\hline 60 & 300 & 27750 & 10699 & 14141 & 7559 & 13610 & 3534 \\
\hline 60 & 450 & 31125 & 19414 & 15688 & 9270 & 15434 & 8360 \\
\hline 60 & 600 & 27178 & 12628 & 13868 & 7614 & 13312 & 5396 \\
\hline 90 & 0 & 14125 & 6494 & 6204 & 3010 & 7921 & 3957 \\
\hline 90 & 150 & 16248 & 7603 & 7614 & 3786 & 8635 & 3977 \\
\hline 90 & 300 & 17921 & 8085 & 8558 & 4338 & 9362 & 3489 \\
\hline 90 & 450 & 16919 & 8646 & 8345 & 4981 & 8573 & 3788 \\
\hline 90 & 600 & 18216 & 9585 & 8987 & 5268 & 9229 & 4329 \\
\hline 120 & 0 & 8550 & 3982 & 4026 & 2128 & 4524 & 1873 \\
\hline 120 & 150 & 13276 & 6282 & 6650 & 3423 & 6625 & 2909 \\
\hline 120 & 300 & 13505 & 4732 & 6383 & 2614 & 7121 & 2184 \\
\hline 120 & 450 & 12979 & 5438 & 5602 & 3518 & 7376 & 3919 \\
\hline 120 & 600 & 11851 & 5276 & 5918 & 2879 & 5932 & 2588 \\
\hline
\end{tabular}


Cuadro 5. Producción anual de materia seca de morera a diferentes frecuencias de corte por niveles de fertilización nitrogenada en kg/ha. Cartago, Costa Rica. 1995.

\begin{tabular}{|c|c|c|c|c|c|c|c|}
\hline \multicolumn{2}{|c|}{ Variable } & \multirow{2}{*}{$\begin{array}{c}\text { Promedio } \\
\text { Planta entera }\end{array}$} & \multirow[t]{2}{*}{ DE } & \multirow{2}{*}{$\begin{array}{l}\text { Promedio } \\
\text { Tallos }\end{array}$} & \multirow[t]{2}{*}{ DE } & \multirow{2}{*}{$\begin{array}{c}\text { Promedio } \\
\text { Hojas }\end{array}$} & \multirow[t]{2}{*}{ DE } \\
\hline $\begin{array}{c}\text { Frecuencias } \\
\text { de corte (días) }\end{array}$ & $\begin{array}{c}\text { Fertilizante } \\
\text { nitrogenado } \\
\text { kg/ha/año }\end{array}$ & & & & & & \\
\hline 56 & 0 & 12027 & 5343 & 5016 & 3163 & 7011 & 3215 \\
\hline 56 & 150 & 13023 & 9975 & 5598 & 3555 & 7425 & 3447 \\
\hline 56 & 300 & 15303 & 7977 & 6436 & 3777 & 8866 & 4285 \\
\hline 56 & 450 & 11348 & 7600 & 4785 & 3414 & 6561 & 3195 \\
\hline 56 & 600 & 13043 & 9298 & 5782 & 4450 & 7261 & 3858 \\
\hline 84 & 0 & 18562 & 6790 & 8663 & 2816 & 9900 & 4044 \\
\hline 84 & 150 & 17833 & 3656 & 8673 & 2288 & 9160 & 1452 \\
\hline 84 & 300 & 18933 & 4462 & 9252 & 2585 & 9680 & 1926 \\
\hline 84 & 450 & 25767 & 11865 & 11673 & 7037 & 14093 & 5712 \\
\hline 84 & 600 & 20975 & 6857 & 10294 & 3239 & 10682 & 3670 \\
\hline 112 & 0 & 21762 & 9375 & 10855 & 5686 & 10904 & 5011 \\
\hline 112 & 150 & 31452 & 10274 & 17501 & 6819 & 13950 & 6359 \\
\hline 112 & 300 & 27153 & 12949 & 15023 & 8100 & 12130 & 4976 \\
\hline 112 & 450 & 28404 & 8560 & 15724 & 6652 & 12678 & 7909 \\
\hline 112 & 600 & 26245 & 13571 & 14602 & 7669 & 11644 & 6024 \\
\hline
\end{tabular}

bajo idénticas frecuencias de corte. Se hace notar en esta comparación la persistrencia del cultivo al cumplir cuatro años de establecido, así como la capacidad de rebrote y la persistencia de la planta sometida a la defoliación periódica para la producción de forraje usado en la alimentación de bovinos y caprinos (Castro, 1989).

En experimentos previos se ha observado que tanto la distancia de siembra como la frecuencia de corte, tienen una gran influencia sobre el rendimiento de la morera (Benavides et al., 1986; Rodríguez et al., 1989 y Boschini et al.,1998). En este experimento se confirma que la distancia de siembra influye el rendimiento de la planta productora de follaje (Cuadro 2). Se observó que a mayor espaciamiento entre plantas disminuye el rendimiento por hectárea. Sin embargo, la producción de biomasa por planta, en forma proporcional, tanto hojas como tallos son estimulados a aumentar su masa al aumentar la distancia entre plantas. Este rendimiento fue de 0,51 y $0,52 \mathrm{~kg} / \mathrm{planta} / \mathrm{año}$ de hojas y tallos en la densidad de $60 \mathrm{~cm}$ entre plantas; 0,71 y 0,71 $\mathrm{kg} /$ planta/año a $90 \mathrm{~cm}$ y de 0,91 y $0,82 \mathrm{~kg} /$ planta/año de hojas y tallos respectivamente en la distancia de $120 \mathrm{~cm}$. Cabe indicar que el rendimiento masal de hojas por planta/año fue completamente lineal al aumentar la distancia de siembra, a razón de $200 \mathrm{~g} / \mathrm{plan}$ ta/año por cada $30 \mathrm{~cm}$ de aumento en la distancia entre plantas. En el tallo ese rendimiento masal tuvo un incremento de $190 \mathrm{~g} / \mathrm{planta} / \mathrm{año}$ al aumentar también 30 $\mathrm{cm}$ en la distancia entre plantas, en el rango de distanciamiento de 60 a $90 \mathrm{~cm}$ y de $110 \mathrm{~g} /$ planta/año al aumentar el distanciamiento $30 \mathrm{~cm}$ adicionales. Esto in- dica que el incremento de tallo por planta disminuye al aumentar el distanciamiento de siembra. Tieng et al. (1988) y Li et al. (1996) han indicado este posible comportamiento al estudiar el rendimiento de la morera bajo diversas distancias de siembra.

Los resultados de producción por área (Cuadro 2) señalan que la morera podría establecerse a distancias de siembra menores a $60 \mathrm{~cm}$ entre plantas, con mayores rendimientos esperados. Sin embargo, claramente se debe esperar un rendimiento anual de hojas y tallos bajo por planta, bajo las condiciones de luminosidad, temperatura, precipitación y suelo de esta zona geográfica. En Turrialba (Benavides, Borel y Esnaola 1986) donde la temperatura ambiental media y la luminosidad a través del año son mayores, se obtuvieron rendimientos de 2,32 kg/planta/año de materia seca con podas a $50 \mathrm{~cm}$ de altura sobre el nivel de suelo y de 2,12 $\mathrm{kg} /$ planta/año con podas a $100 \mathrm{~cm}$ de altura en cultivos de 20.000 plantas/ha. Estos valores de crecimiento acumulado por planta fueron mayores a los obtenidos en este experimento; sin embargo, coinciden con los resultados informados por Espinoza (1996) para las zonas de Puriscal y Coronado.

La intensidad de la defoliación de la planta de morera tuvo un marcado efecto $(\mathrm{P}<0,01)$ sobre el rendimiento de biomasa en hojas y tallos. A mayor intervalo de corte se observaron mayores rendimientos (Cuadro 2), lo cual está fisiológicamente relacionado con el periodo de rezago entre corte y rebrote, la reserva de nutrimentos al momento de la poda, la tasa 
potencial de crecimiento de la planta y la prevalencia de condiciones climáticas favorables (Taiz et al., 1991). Cada intervalo de poda arrastró en forma sistemática el efecto residual del estrés producido por la poda anterior. Los resultados muestran claramente que la producción anual por hectárea fue mayor a intervalos largos de poda y disminuyó en forma proporcional en los intervalos de defoliación cortos. Similares respuestas encontraron Rodríguez, Arias y Quiñones (1994) y Boschini, Dormond y Castro (1998) al estudiar el efecto de las frecuencias de corte a través de los distintos periodos del año en Guatemala y Costa Rica, respectivamente. La producción de hojas y tallos, separadamente, tuvieron un comportamiento diferente a la suma de ellos. La producción de hojas en los dos primeros intervalos de poda fue superior al observado en el tallo. A 112 días, los rendimientos se invirtieron, siendo mayor la producción de tallo que de hoja. Entre 84 y 112 días se observa que la hoja tiene una tasa de crecimiento diario de 55,6 kg/ha y el tallo de $179 \mathrm{~kg} / \mathrm{ha}$. En este intervalo de desarrollo de la planta, la masa diaria relativa de tallo crece cerca de tres veces más rápido que la masa diaria relativa de hoja. Se estimó que a 98 días de rebrote, se produce el punto de intersección. A partir de esta edad, la planta comienza a adquirir las características leñosas naturales de una especie arbórea. A edades mayores, se complica el manejo de la planta y se pierde interés de aprovecharse como alimento forrajero. Este punto de intersección, había sido informado anteriormente por Boschini, Dormond y Castro (1998) a los 100 días de crecimiento, bajo similares condiciones. Es probable que el cultivo de morera en un ambiente mucho más cálido, la producción de tallo se estimula más temprano y alcanza a la producción de hoja en un periodo menor. Así lo sugieren los resultados de Espinoza (1996) obtenidos en un ambiente tropical seco.

La combinación de las variables distancia de siembra y frecuencia de corte fueron los factores más relevantes sobre la producción de biomasa total, hojas y tallos. El análisis simultáneo de las Figuras 2 y 3 indica que a la distancia de $60 \mathrm{~cm}$ entre plantas hay mayor producción de hojas que tallos hasta los 84 días de crecimiento. Después de esa edad se favorece la acumulación de tallo. En la distancia de $90 \mathrm{~cm}$ entre plantas se mantiene aceptable el rendimiento de hojas y tallos en cualquier edad hasta los 112 días. Sin embargo, cuando las plantas se establecieron a $120 \mathrm{~cm}$ de distancia entre plantas, la proporción de hojas y tallos acumulada hasta los 84 días podría ser la edad máxima de corte. Boschini, Dormond y Castro (1998) encontraron que el rendimiento de hoja y tallo decaía a los 112 días cuando se establecía a $120 \mathrm{~cm}$ entre plantas y contrariamente a estos resultados, no recomendó el manejo de podas a 112 días en plantaciones sembradas a distanciamientos de $60 \mathrm{~cm}$ entre plantas.
La falta de respuesta de la morera a los diferentes niveles de fertilización nitrogenada en este experimento, es contrario a lo reportado en la literatura (IFA, 1992; Ting-Zing et al., 1988; Rodríguez, Arias y Quiñones, 1994; Benavides, Lachaux y Fuentes, 1994). La aplicación de fertilizante en este cultivo debe tomar en consideración la fisiología de la planta y las características físicas y químicas del suelo. La planta pasa por tres estados fisiológicos. El primero es de crecimiento inicial, con emergencia de brotes foliares y el desarrollo de ramas. Se alimenta de los nutrientes almacenados en raíz y el tronco. La absorción radical de fertilizante es de mantenimiento basal. El segundo periodo, es de crecimiento acelerado, caracterizado por una máxima asimilación de fertilizantes aplicados. Se observa la mayor producción foliar. Posteriormente, viene un periodo de almacenamiento, se reduce la elongación y se acumularon los nutrimentos asimilados o transformados, particularmente en ramas y en la raíz. En este estado se maximiza la producción de tallo y la acumulación de carbono (Cardin y Abramides, 1972; Ayuzawa, 1972).

En Costa Rica, Espinoza (1996), trabajando en tres zonas diferentes con tres variedades de morera y niveles de fertilización nitrogenada $(180,360$ y 540 kg/ha/año) encontró respuestas diferenciadas en la producción de biomasa total. Con la aplicación de $180 \mathrm{~kg}$ de N/ha/año obtuvo un rendimiento medio de 16,1 t/ha/año con $360 \mathrm{~kg}$ de N/ha/año fue de 21,6 t/ha/año y con 540 la producción anual fue de 24,1 t/ha. Estos rendimientos no se alejan mucho de los obtenidos en el presente experimento, pero si describen una curva de rendimientos decrecientes típico de los ensayos de fertilización. Rodríguez, Arias y Quiñones (1994) trabajando con frecuencias de poda y niveles de fertilización nitrogenada también reportaron una respuesta positiva. Benavides, Lachaux y Fuentes (1994) señalan que la respuesta se acentúa conforme el intervalo de poda es mayor dentro del rango de seis a 12 semanas entre cortes.

Tanto Espinoza (1996) como Rodríguez, Arias y Quiñones (1994) y Benavides, Lachaux y Fuentes (1994), en los reportes efectuados, no hicieron referencia a la relación entre el contenido de nitrógeno en los suelos empleados y la respuesta de la producción obtenida. Se presume que trabajaron en cultivos de morera sobre suelos pobres en nitrógeno. En el presente experimento, el cultivo de morera estuvo sembrado en un suelo con un contenido de $\mathrm{N}$ relativamente alto (Cuadro 1 ), con un valor medio de $0,29 \%$ de $\mathrm{N}$ total en los primeros $30 \mathrm{~cm}$ de suelo. Adicionalmente, se observa que los valores de materia orgánica son altos, y los contenidos de nitrógeno total, están en proporción directa al contenido de materia orgánica presente en cada profundidad muestral de suelo. Se observa que la retención de 
nitrógeno es alta en consecuencia. Bertsch, 1995, describe que el rango normal del nitrógeno total en el suelo va de 0,02 a $0,40 \%$ y que más allá de ese límite, sólo ocurre en presencia de altos contenidos de materia orgánica. En el suelo del cultivo experimental, se observó un valor de $0,46 \%$ de nitrógeno total en la profundidad de 10 a $20 \mathrm{~cm}$. En el Cuadro 1, se observan valores altos de nitrógeno soluble, tanto en forma de amonio como de nitrato al inicio del trabajo. La falta de respuesta de la morera a la fertilización nitrogenada en el presente experimento, se explica por los altos niveles de nitrógeno presentes en el suelo, tomando en cuenta también que el contenido de los restantes nutrimentos minerales del suelo estuvieron presentes en cantidades suficientes, dentro del rango medio de fertilidad, para no calificar a éste, como otro posible factor de limitación de la producción de biomasa en el experimento.

\section{CONCLUSIONES Y RECOMENDACIONES}

Los resultados mostraron un aumento en el rendimiento anual de biomasa total por unidad de área conforme disminuyeron las distancias de siembra entre plantas. La competencia que cada planta tuvo en el área basal, para su desarrollo radical y foliar, se manifestó en una disminución del rendimiento individual por cada planta al aumentar la densidad de siembra.

La producción de biomasa en hojas y tallos se incrementó en la medida que aumentó el largo del intervalo de poda, encontrándose una mayor proporción masal de hojas. Sin embargo, se manifestó un desmejoramiento en la relación de hoja:tallo al aumentar el largo del intervalo de corte. Se estimó que a 98 días de edad de rebrote, esta relación disminuyó a uno y posteriormente, la planta de morera adquirió una formación leñosa indeseable que creció cada día, perdiendo aceleradamente su excelente valor como productora de forraje arbustivo.

Los niveles de nitrógeno aplicados al suelo no mostraron respuestas diferenciales sobre la producción de biomasa total, ni se evidenció alguna alteración importante en las proporciones estructurales de la planta que reflejara un cambio en el rendimiento de hojas y tallos. La falta de respuesta se explicó por los altos contenidos de nitrógeno y materia seca presentes en el suelo al inicio del experimento. Se estima que con niveles de nitrógeno total en el suelo superiores a $0,29 \%$ o contenidos de nitrógeno amoniacal y de nitrato mayores a $24 \mathrm{mg} / \mathrm{kg}$ de suelo, no se recomienda la aplicación de fertilizante nitrogenado.

\section{LITERATURA CITADA}

A.O.A.C. 1980. Methods of analysis. Edicion 13. Washington. D.C. EUA. Association of Official Analysis of Chemistry.

AYUZAWA, CH, 1972. Handbook of silkworm rearing. Fuji. Pum. Ltd. Tokio. No 1. p 319.

BENAVIDES, J., BOREL, R.; ESNAOLA, M.A. 1986. Evaluación de la producción de forraje del árbol de Morera (Morus sp) sometido a diferentes frecuencias y altura es de corte. Centro Agronómico Tropical de Investigación y Enseñanza. Resumen de las investigaciones realizadas con rumiantes menores, en el Proyecto de Sistemas de Producción Animal. Serie Técnica. Informe Técnico No 67. pp. 74-76.

BENAVIDES, J. 1986. Efecto de diferentes niveles de suplementación con follaje de Morera (Morus sp) sobre el crecimiento y consumo de corderos alimentados con pasto (Pennisetum purpureum). Resumen de las investigaciones realizadas con rumiantes menores, cabras y ovejas. Proyecto de Sistemas de Producción Animal. CATIE. Serie Técnico. Informe Técnico No 67. pp. 74-76.

BENAVIDES, J.; LACHAUX, M.; FUENTES, M. 1994. Efecto de la aplicación de estiércol de cabra en el suelo sobre la calidad y producción de biomasa de Morera (Morus sp). Arboles y arbustos forrajeros en América Central. Serie Técnica. CATIE. Informe Técnico No 236. Volumen II. pp. 495-514.

BERTSCH, F. 1995. La fertilidad de los suelos y su manejo. Asociación Costarricense de la Ciencia del Suelo. San José, Costa Rica. 157 p.

BLANCO, R. 1992. Distancia de siembra y altura de corte en la producción y calidad del forraje de Morera (Morus sp) en el parcelamiento Cuyunta, Escuintla, Guatemala. Guatemala. Universidad de San Carlos. 15 p.

BOSCHINI, C.; DORMOND, H.; CASTRO, A. 1998. Producción de biomasa de la morera (Morus alba) en la Meseta Central de Costa Rica, establecida y cosechada a diferentes distancias de siembra, alturas y frecuencias de poda. Revista Agronomía Mesoamericana 9(2):28-39.

CARDIN, A.O.; ABRAMIDES, P. 1972. Técnica de cultura de la morera Morus alba. Boletín Técnico No 3. 28 p.

CASTRO, A. 1989. Producción de leche de cabras alimentadas con King grass (Pennisetum purpureum $\mathrm{x}$ P. typloides), suplementales con diferentes niveles de follaje de Poró (E. poeppigrama) y de fruto de plátano va de $(\mathrm{Mu}$ $s a$ sp. var. Pelipita). Tesis M.Sc. Turrialba, C.R. UCR/CATIE. 58 p.

ESPINOZA, E. 1996. Efecto del sitio y del nivel de fertilización nitrogenada sobre la producción y calidad de tres 
variedades de morera en Costa Rica. Tesis de Maestría. CATIE. Turrialba, Costa Rica. 72 p.

ESTRADA, A. 1995. Efecto de la sustitución de King Grass por morera sobre los parámetros de degradación y fermentación ruminal de cuatro forrajes de calidad constante. Tesis de Maestría. CATIE. Turrialba, Costa Rica. $63 \mathrm{p}$.

GONZÁLEZ, J. 1996. Evaluación de la calidad nutricional de la morera fresca y ensilada con bovinos de engorde. Tesis de Maestría. CATIE. Turrialba, Costa Rica. 67 p.

IFA. INTERNATIONAL FERTILIZER INDUSTRY ASSOCIATION. 1992. IFA World fertilizer use manual. Mulberry Chart. BASF. Aktiengesells- Chaft. Agricultural Research Station. Germany. pp. 595-601.

LIN, J.T.; YU, S.J.; HSIEH, F.K. 1994. Effects of plant spacing on the yield and chemical compositions of mulberry leaves. Journal of the Agricultural Association of China. 167:43-49.

PAOLIERI, L. 1970. Competicao de variedades de Amoreiras. Boletín Técnico de Sericultura No 57. Secretaría de Agricultura. Coordinadora de Pesquiza Agropecuaria. Instituto de Zootecnia. Secao de Sericultura. pp. 3-16.

RODRÍGUEZ, C.; ARIAS, R.; QUIÑONES, J. 1994. Efecto de la frecuencia de poda y el nivel de fertilización ni- trogenada, sobre el rendimiento y calidad de la biomasa de Morera (Morus sp) en el trópico seco de Guatemala. Arboles y arbustos forrajeros en América Central. Serie Técnica. CATIE. Informe Técnico No 236. Volumen II. pp. 515-528.

SAS. 1985. Statistical analysis system. SAS User’s Guide: Statistics (Version 5 Ed.) SAS Institute Inc. Cary, NC. sp.

TAIZ, L.; ZEIGER, E. 1991. Plant physiology. The Benjamin/Cummings Publishing Company, Inc. California, USA. sp.

TING-ZING, Z.; YUN-FAN, T.; GUANG-XIEN, H.; HUAIZHONG, F.; BEN, MA. 1988. Mulberry cultivation. FAO agricultural services Bulletin 73/1. Food and Agricultural Organization of the United Nations. Rome. p. 127.

VASQUEZ, A. 1982. Estudio detallado de los suelos de la Estación Experimental de Ganado Lechero El Alto. Escuela de Fitotecnia, Facultad de Agronomía, Universidad de Costa Rica. p. 36.

VELAZQUEZ, C.M. 1992. El forraje de morera (Morus sp) como fuente de proteina en dietas a base de ensilado de sorgo (Sorghun bicolor x Sorghum sudanense) para novillos en el parcelamiento Cuyutá. Tesis de Licenciatura. Universidad de San Carlos, Guatemala. 43 p. 